\title{
Symmetry Driven Photonics for New Active Functionality On-Chip
}

\author{
Liang Feng \\ University of Pennsylvania, USA \\ fenglia@seas.upenn.edu
}

\begin{abstract}
Symmetry and topology are fundamental notions existing in all kinds of natural systems, from spiral galaxies and hurricanes to amino acids in molecules and non-trivial topologically protected electronic states in condensed matter. A stream of photons is typically topologically trivial, nevertheless, its full-vector nature intrinsically endows light with full capability of creating and carrying unique symmetry and topology, especially non-Hermitian symmetries that cannot be easily implemented in condensed matter. Explorations of symmetry and topology on a photonic platform not only deepen our understanding of fundamental physics, but also enable novel material properties to facilitate technological breakthroughs for photonic applications. In this seminar, I will present our recent efforts on investigating the non-Hermitian parity-time symmetry, supersymmetry, and topological physics for the next generation of optical communication and information technology. We demonstrated an orbital angular momentum (OAM) microlaser that structures and twists the lasing radiation at the microscale, which can provide an additional OAM-based information dimension to meet the growing demand for information capacity. By strategically interfacing non-Hermitian photonic materials and topological physics, we realized the dynamic control of robust topological transmission links of light inside the bulk of a photonic topological insulator, routing optical signals in a highly flexible and scalable manner. Furthermore, we conducted a supersymmetry-based formalism to achieve higher-dimensional microlaser phaser arrays producing high-radiance, small-divergence laser beams with orders of magnitude enhancement in energy density.
\end{abstract}

